\section{Alelos del complejo principal de histocompatibilidad (HLA) en pacientes con trastorno bipolar en Turquía}

Se puede observar diferencias étnicas significativas desde el punto de vista de los marcadores biológicos en diferentes enfermedades psiquiátricas. El sistema HLA, que es un marcador genético en los trastornos del estado de ánimo, es un caso de este tipo. La investigación relacionada indica que los genes situados en la región del HLA del sexto cromosoma pueden ser uno de los diversos factores que contribuyen al origen de los trastornos del estado del ánimo. Se ha comunicado que hay un aumento significativo en las frecuencias de HLA B16, HLA A29 y B21, HLA-B7 en los pacientes con trastornos bipolares $[1,4,5]$. Sin embargo, otros autores no han observado una relación entre los haplotipos del HLA y el trastorno del estado de ánimo [3, 6]. Investigamos la relación entre el trastorno bipolar del estado de ánimo y los antígenos del HLA estudiando un grupo bastante grande y étnicamente homogéneo.

Se incluyó en el estudio a 50 pacientes ( 24 mujeres y 26 hombres) diagnosticados como casos de trastorno bipolar del estado de ánimo de tipo I según los criterios del DSMIV. La cantidad media de episodios maníacos y de depresión era $4,2(\mathrm{DT}=1,4)$ y $2,1 \quad(\mathrm{DT}=1,1)$, respectivamente. Además, se formó un grupo de control de 100 personas sin enfermedades mentales anteriores o actuales sometidas a exploración como donantes para trasplante de riñón. Se obtuvo el consentimiento informado escrito de todos los sujetos. No hubo diferencias entre los dos grupos desde el punto de vista del sexo y la edad.

Las muestras de sangre se analizaron según el método de microlinfocitotoxicidad [2]. Los loci examinados eran HLA A (A1-3, A11, A23-26, A28-34, A43, A66); B (B7, B8, $\mathrm{B} 13, \mathrm{~B} 14, \mathrm{~B} 18, \mathrm{~B} 27, \mathrm{~B} 35, \mathrm{~B} 37-42$, B44-47, B49-59, B62, B63, B67, B70, B73); C (Cw1, Cw3-6); DR (DR1, DR1518, DR4, DR11-14, DR7-10, DR51-53, DQ1, DQ2, DQ4, DQ7). Los antígenos DQ8 y DQ9 del HLA no se pudieron examinar serológicamente. Todos los pacientes y los controles eran de raza blanca y de origen turco. En la comparación intergrupos, se encontró que los antígenos HLA-B7 $\left(\chi^{2}=3,64, \mathrm{gl}=1, \mathrm{p}=0,05\right)$, HLA-DR $11\left(\chi^{2}=4,83, \mathrm{gl}=\right.$ $1, \mathrm{p}=0,04)$ y HLA-DQ7 $\left(\chi^{2}=5,39, \mathrm{gl}=1, \mathrm{p}=0,03\right)$ eran más frecuentes en el grupo de pacientes bipolares. Sin embargo, estos resultados no eran significativos ya después de corregir el número de alelos. Nuestros hallazgos indican que los alelos del HLA pueden no conferir susceptibilidad al trastorno bipolar en la población turca.

\section{Bibliografía}

[1] Biederman J, Keller M, Lavori P, Harmatz J, Knee D, Dubey D, et al. HLA haplotype A26-B38 in affective disorders: lack of association. Biol Psychiatry 1987;22:221-4.

[2] Jun TY, Pae CU, Chae JH, Pyo CW, Han H. Human leukocyte antigen alleles in patients with bipolar disorder in the Korean population. Psychiatry Clin Neurosci 2002;56:453-7.

[3] Ozcan E, Taskin R, Banoglu R, Babacan R, Tuncer E. HLA antigens in schizophrenia and mood disorders. Biol Psychiatry 1996;39:8915 .

[4] Sorokina TT, Evsegneev RA, Levin VI; Semenov GV. Features of the distribution of HLA-antigens among patients with endogenous psychosis. Zh Nevropatol Psikhiatr Im S S Korsakova 1987;87:8858 [in Russian].

[5] Terasaki PI. Techniques in HLA typing. In: Brand DL, Rae JG, editors. Manual of tissue tuping techniques. Bethesda: National Institute of Health; 1970.p. 122-4.

[6] Ventura T, Lobo A, Marco J. HLA antigens in bipolar affective patients. Actas Luso Esp Nerol Psiquatr Cienc Afines 1990;18:33943 [in spanish]

Alp Ü̧̧ok

Facultad de Medicina de Estambul, Departamento de Psiquiatría,

Millar street, Capa, 34390 Estambul, Turquia Correo electrónico: alpucok@superonline.com

(A. Üçok).

Ugur Akar

Facultad de Medicina de Estambul, Departamento de Biología Médica, Estambul,

Turquía.

Aslihan Polat, Olcay Yazici

Facultad de Medicina de Estambul, Departamento de Psiquiatria, Millet street, Capa, 34390 Estambul, Turquia. 